\title{
Analysis of blood flow in pulmonary hypertension with the pulsed Doppler flowmeter combined with cross sectional echocardiography
}

\author{
MITSUNORI OKAMOTO, * KUNIO MIYATAKE, $†$ NAOKAZU KINOSHITA,† \\ HIROSHI SAKAKIBARA, † YASUHARU NIMURA * \\ From the ${ }^{\star}$ Research Institute and the $†$ Division of Cardiology, National Cardiovascular Center, Osaka, fapan
}

SUMMARY Blood flow patterns were analysed at nine points in the pulmonary area using the pulsed Doppler technique combined with cross sectional echocardiography in 53 patients with heart disease and 10 healthy subjects. In subjects with a normal pulmonary artery pressure the blood flow pattern in systole showed a gradual acceleration and deceleration with a rounded summit in mid systole, designated the round type. In patients with pulmonary hypertension it showed a rapid acceleration and early deceleration with a sharp peak in early systole, designated the triangular type. The acceleration time index, defined as the ratio of the time interval from the beginning to the peak of ejection to the ejection time, showed a significant inverse correlation with mean pulmonary artery pressure. In pulmonary hypertension a prominent reverse flow occurred in the right posterior part of the pulmonary trunk during mid-systole and early diastole, indicating the presence of a vortex. Similar flow patterns were also seen in patients with idiopathic pulmonary artery dilatation. The factors responsible for the triangular type were principally the reduced capacitance and increased impedance of the pulmonary vascular tree. Those responsible for the reverse flow were the curved path of the blood flow and the dilatation of the pulmonary artery.

The development of the pulsed Doppler ultrasound technique has provided a non-invasive method of examining the blood flow in deep lying blood vessels and cardiac chambers. The usefulness of this technique in detecting abnormal flow in valvular and congenital heart disease has been well established. ${ }^{1-18}$ In this study we analysed the blood flow pattern in the pulmonary artery with special reference to changes in pulmonary hypertension using this technique.

\section{Patients and methods}

Twenty four patients with aortic and mitral valve disease and 26 with a secundum atrial septal defect were studied. Three patients with idiopathic pulmonary artery dilatation were also studied for comparison. Their ages ranged from 20 to 74 (mean 40.6) years. The diagnoses were confirmed by cardiac catheterisa-

Requests for reprints to Dr Mitsunori Okamoto, Research Institute, National Cardiovascular Center, 125 Fujishirodai 5-chome, Suita, Osaka 565, Japan.

Accepted for publication 27 October 1983 tion and angiography. Mean pulmonary artery pressure was higher than $25 \mathrm{~mm} \mathrm{Hg}$ in 23 of the 50 patients, and none of three with idiopathic pulmonary artery dilatation had pulmonary hypertension. Ten healthy subjects were also examined as controls.

\section{EQUIPMENT}

The system used consisted of a directional pulsed Doppler flowmeter (Hitachi Medico EUD-4Z) and a commercially available electrocardiogram gated compound cross sectional echocardiograph based on the stop motion principle (Aloka SSD-120). Details of this system have been previously described.$^{8} \mathrm{~A}$ transducer attached to a guide arm to indicate the position and the angle of the transducer was used for both the Doppler flowmeter and the echocardiograph by switching the mode of display (Fig. 1). The ultrasound frequency of the Doppler flowmeter was $2.5 \mathrm{MHz}$ and the pulse repetition rate $4.5 \mathrm{kHz}$ or $6.3 \mathrm{kHz}$. The size of the sample volume was $6 \mathrm{~mm}$ (width) $\times 5 \mathrm{~mm}$ (depth). The frequency spectrum of the Doppler signals was analysed with a real time 

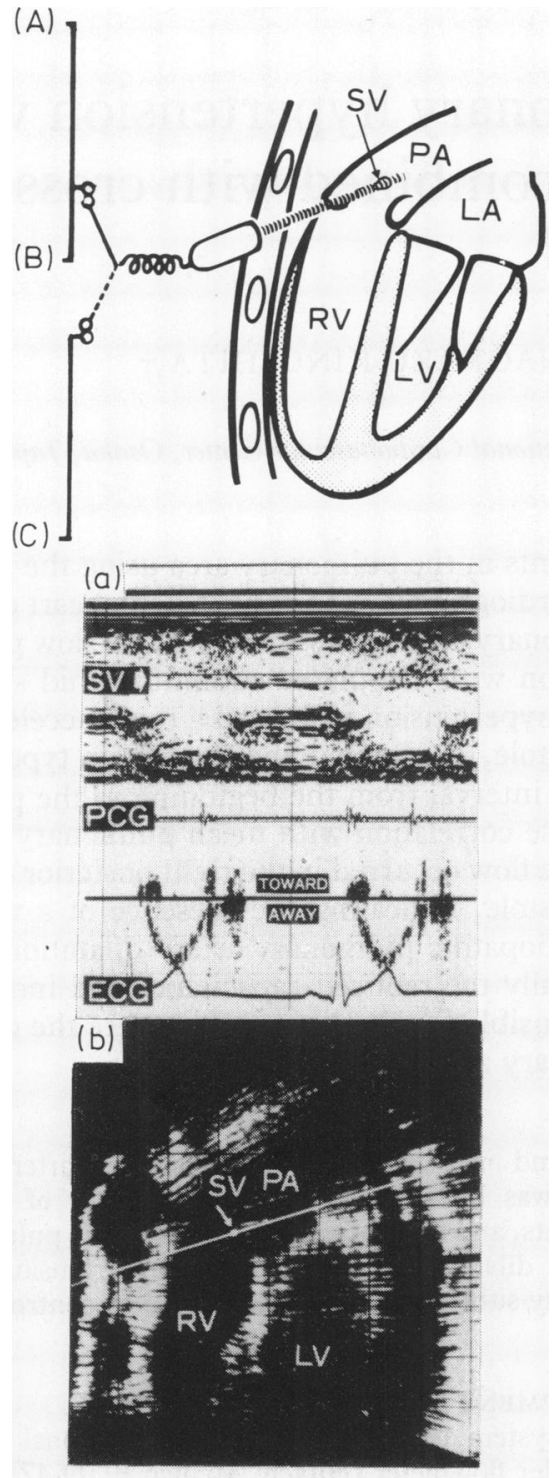

Fig. 1 Diagram of the combined system using an $M$ mode echocardiograph $(A)$, an ultrasonic pulsed Doppler flowmeter $(B)$, and an electrocardiogram-gated cross sectional echocardiograph $(C)$. (a) Doppler signals polygraphically displayed on an $M$ mode echocardiogram by the same ultrasound beam. (b) Cross sectional echocardiogram by a contact compound scanning showing a sagittal section of the heart from the right ventricular outflow tract to the pulmonary trunk; the bright line and dot on it indicate the beam direction for Doppler recording and the sampling site respectively. ECG, electrocardiogram; $L A$, left atrium; $L V$, left ventricle; $P A$, pulmonary artery; $P C G$ phonocardiogram; $R V$, right ventricle; $S V$, sample volume. sound spectrograph (Hitachi Medico EUD-4Z). After the imaging of a cross sectional echocardiogram by compound scanning the direction of the ultrasound beam for the Doppler recording and the sampling site of the Doppler signals were displayed by a bright line and a bright spot respectively superimposed on the cross sectional image. The Doppler signals were then recorded.

In five patients with pulmonary hypertension another Doppler device (Toshiba prototype or SDS$10 \mathrm{~A}$ ) incorporated in a wide angle phased array echograph (Toshiba Sonolayergraph SSH-11A) was also used. With this system the real time cross sectional image of the heart and the Doppler signals can be obtained simultaneously. The ultrasound frequency was $2.4 \mathrm{MHz}$ and the pulse repetition rate $4 \mathrm{kHz}$ or $6 \mathrm{kHz}$. Analysis of the frequency of the Doppler signals was based on the principle of fast Fourier transform. The size of the sample volume was $4 \mathrm{~mm}$ (width) $\times 2 \mathrm{~mm}$ (depth).

The Doppler signals were displayed simultaneously with the electrocardiogram, phonocardiogram, and $M$ mode echocardiogram using a strip chart recorder (Electronics For Medicine VR-12 and Toshiba LSR$20 \mathrm{~A}$ ) at a paper speed of $50 \mathrm{~mm} / \mathrm{s}$. Flow velocity components towards and away from the transducer were displayed above and below the baseline respectively.

\section{RECORDING AND ANALYSIS OF BLOOD FLOW PATTERN}

The patient was examined supine. The sagittal cross section of the heart including the right ventricular outflow tract and the pulmonary trunk was imaged with the transducer placed at the second or third left intercostal space. The Doppler beam was directed successively into the anterior, central, and posterior parts of the pulmonary area (Fig. 2). Blood flow signals were recorded in each direction at the right ventricular outflow tract near the pulmonary valve, the pulmonary orifice, and the pulmonary trunk near the pulmonary valve. The horizontal cross section at the level of the pulmonary trunk was also used for further analysis of the blood flow in the pulmonary trunk.

In all subjects the blood flow pattern in the central part of the pulmonary area was classified into three types for the purpose of analysis (see results). The observations were performed independently by three observers, the final classification being based on the agreement of at least two of the three observers.

To determine the timing of the peak flow velocity the acceleration time index was measured in the central part of the pulmonary orifice using the flow pattern. The index was defined as the ratio of the time interval from the beginning of ejection to the time of the peak flow velocity-that is, the acceleration time- to the ejection time (Fig. 3). A decrease in the 


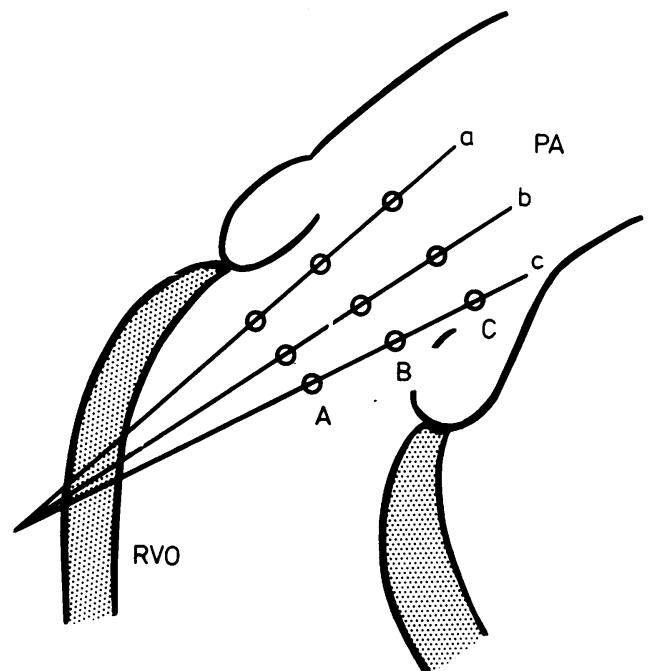

Fig. 2 Diagram of the direction of the ultrasound beam and the sites of sampling for Doppler recording showing a sagittal section of the right ventricular outflow (RVO) tract and the pulmonary trunk viewed from the left. The ultrasound beam was aimed to the anterior (a), central (b), and posterior (c) parts of the pulmonary area. Doppler signals were recorded in the right ventricular outflow tract $(A)$, pulmonary orifice $(B)$, and pulmonary trunk $(C)$ in each beam direction. $P A$, pulmonary artery.

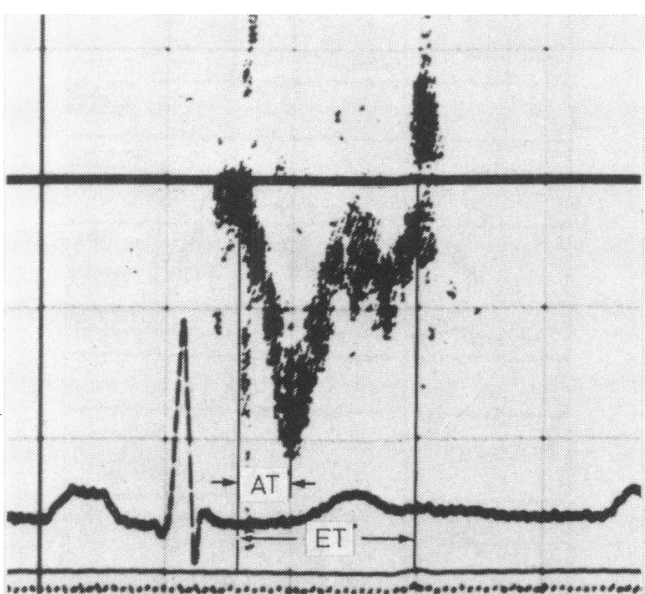

Fig. 3 Measurement of acceleration time index calculated as acceleration time $(A T)$ divided by ejection time $(E T) \times 100 \%$. $A T$, time interval from the beginning of ejection to the time of peak flow velocity; ET, time interval from the onset to the termination of ejection.

index indicates that the peak flow velocity and the deceleration of flow began earlier.

STATISTICS

Differences in mean pulmonary artery pressure and total pulmonary vascular resistance between groups of

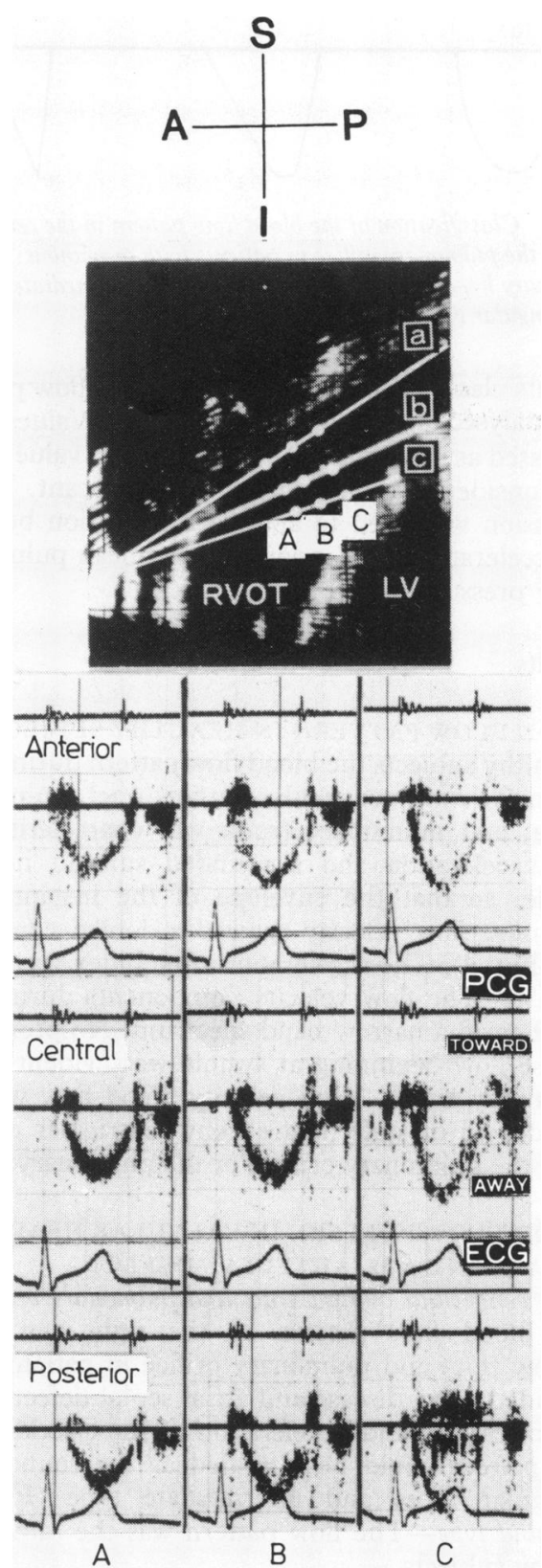

Fig. 4 Doppler recording and cross sectional echocardiogram showing blood flow patterm in a healthy subject in the right ventricular ouffow tract $(A)$, pulmonary orifice $(B)$, and pulmonary trunk $(C)$. Gradual acceleration and deceleration during ejection occurred with the peak flow velocity in mid-systole, showing a round configuration as a whole. There was no prominent reverse flow in the pulmonary trunk. $A$, anterior; $I$, inferior; $P$, posterior, $R V O T$, right ventricular ouflow tract, $S$, superior. 
(a)

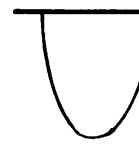

Fig. 5 Classification of the blood flow pattern in the central part of the pulmonary orifice in patients with or without pulmonary hypertension; (a) round type; (b) intermediate type, (c) triangular type.

patients classified according to the blood flow pattern was analysed using an unpaired $t$ test. Values were expressed as means $( \pm$ SD). A probability value $<0.05$ was considered to be statistically significant. Linear regression was used to analyse the relation between the acceleration time index and the mean pulmonary artery pressure.

\section{Results}

BLOOD FLOW PATTERN IN HEALTHY SUBJECTS In healthy subjects the blood flow pattern during ejection in the right ventricular outflow tract, pulmonary orifice, and pulmonary trunk was characterised by slow acceleration and a rounded summit in midsystole, so that the envelope of the instantaneous maximum flow velocity showed a domed configuration (Fig. 4). The acceleration time index was $46 \cdot 2 \%$ $( \pm 3 \cdot 1 \%)$. The flow velocity components during systole showed a narrow band spectrum. No prominent reverse flow beginning at systole was evident in the pulmonary trunk. High velocity blood flow was not recorded in diastole in the right ventricular outflow tract, the pulmonary orifice, or the pulmonary trunk.

\section{BLOOD FLOW PATTERN IN VALVULAR HEART DISEASE AND ATRIAL SEPTAL DEFECT}

Right ventricular outflow tract and pulmonary orifice The blood flow pattern in the right ventricular outflow tract and pulmonary orifice in patients with left sided valve disease and atrial septal defect varied in acceleration and deceleration of the ejection flow. The pattern could be classified as the round type, triangular type, and intermediate type (Fig. 5).

Round type-The flow pattern was the same as in healthy subjects.

Triangular type-In this type the slope of the acceleration phase was more steep and the deceleration occurred earlier than in the round type (Fig. 6). The flow velocity reached a sharp peak in early systole. In a few patients, a reacceleration of flow velocity occurred in late systole.

Intermediate type-This type belonged to neither the round nor the triangular type and showed a relatively rounded peak in early systole.
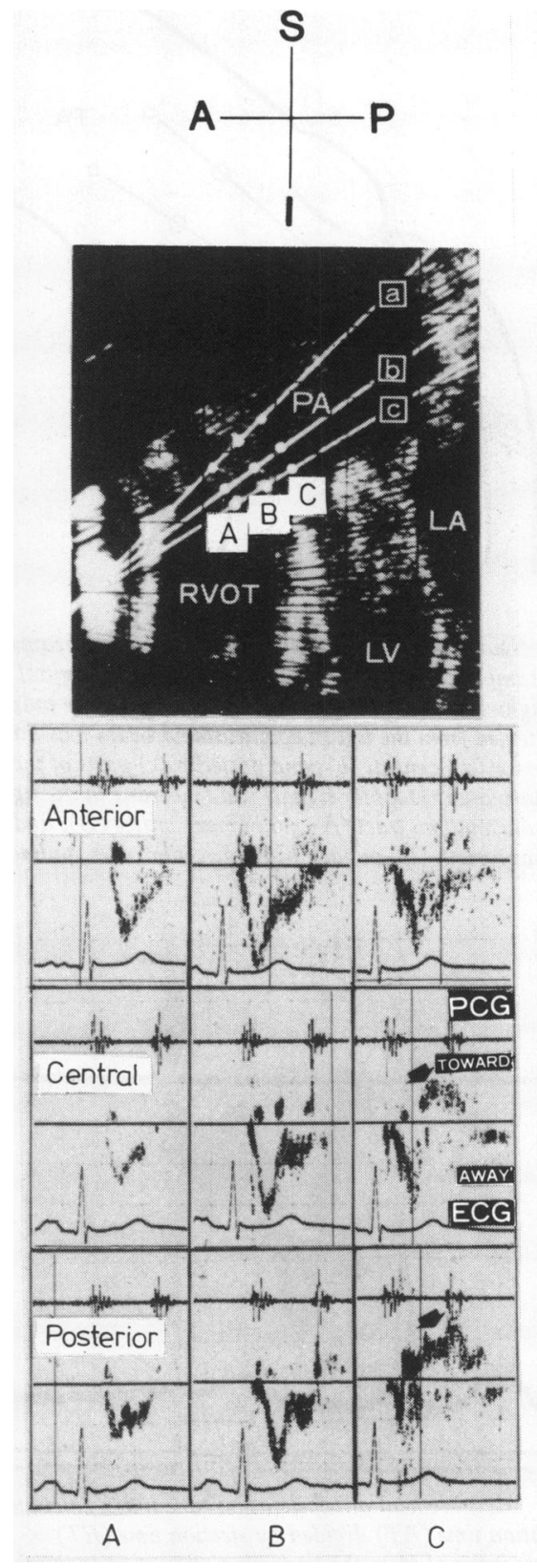

Fig. 6 Doppler recording and cross sectional echocardiogram showing the blood flow pattern in a patient with pulmonary hypertension in the right ventricular oufflow tract $(A)$, pulmonary orifice $(B)$, and pulmonary trunk $(C)$. The triangular flow pattern is present in the right ventricular outflow tract and pulmonary orifice. A prominent reverse flow beginning from mid-systole is evident in the central and posterior parts of the pulmonary trunk (arrows). 
Twenty three of the 50 patients showed the round type, 14 the triangular type, and 13 the intermediate type.

The acceleration time index ranged from 41.3 to $55.7 \%$ (mean $46.5 \pm 3.4 \%$ ) in the round type, from 25.2 to $39.5 \%$ (mean $30.2 \pm 3.8 \%$ ) in the triangular type, and from 30.7 to $46.2 \%$ (mean $37.7 \pm 4.3 \%$ ) in the intermediate type. The index in the triangular type was significantly lower than in the intermediate and round types $(p<0.001)$.

\section{Pulmonary trunk}

In 14 of the 23 patients with the round type technically adequate blood flow signals could be obtained in the pulmonary trunk. In the remaining nine patients the pulmonary trunk could not be satisfactorily visualised on the echocardiogram, so the adequate blood flow signals for analysis could not be obtained. In the 14 patients with the round type the blood flow pattern in the pulmonary trunk was almost similar to that in the pulmonary orifice and showed no prominent reverse flow in systole. In the 14 patients with the triangular type the pattern in the pulmonary trunk varied in relation to the site of the Doppler recording (Fig. 6). In the anterior part of the pulmonary trunk the pattern was similar to that in the pulmonary orifice. In the central and posterior parts of the pulmonary trunk the forward ejection flow ended in early or mid-systole and was followed by a reverse flow which lasted to early or mid-diastole. The reverse flow showed a relatively narrow band velocity spectrum. In 10 of the 13 patients with the intermediate type the pattern in the pulmonary trunk was similar to that of the round type and in the remaining three similar to that of the triangular type.

When examined in the horizontal section at the level of the pulmonary trunk in five patients with pulmonary hypertension with the triangular type the reverse flow was also evident in the right posterior half of the pulmonary trunk but not in the left anterior half (Fig. 7).

\section{BLOOD FLOW PATTERN IN IDIOPATHIC \\ PULMONARY ARTERY DILATATION}

In the three patients with idiopathic pulmonary artery dilatation the blood flow pattern in the right ventricular outflow tract and the pulmonary orifice showed the round, triangular, and intermediate types respectively (Fig. 8). The acceleration time index was $43.8 \%$ in the round type, $30.3 \%$ in the triangular type, and $35.7 \%$ in the intermediate type. In the pulmonary trunk a similar reverse flow was evident in all three patients.
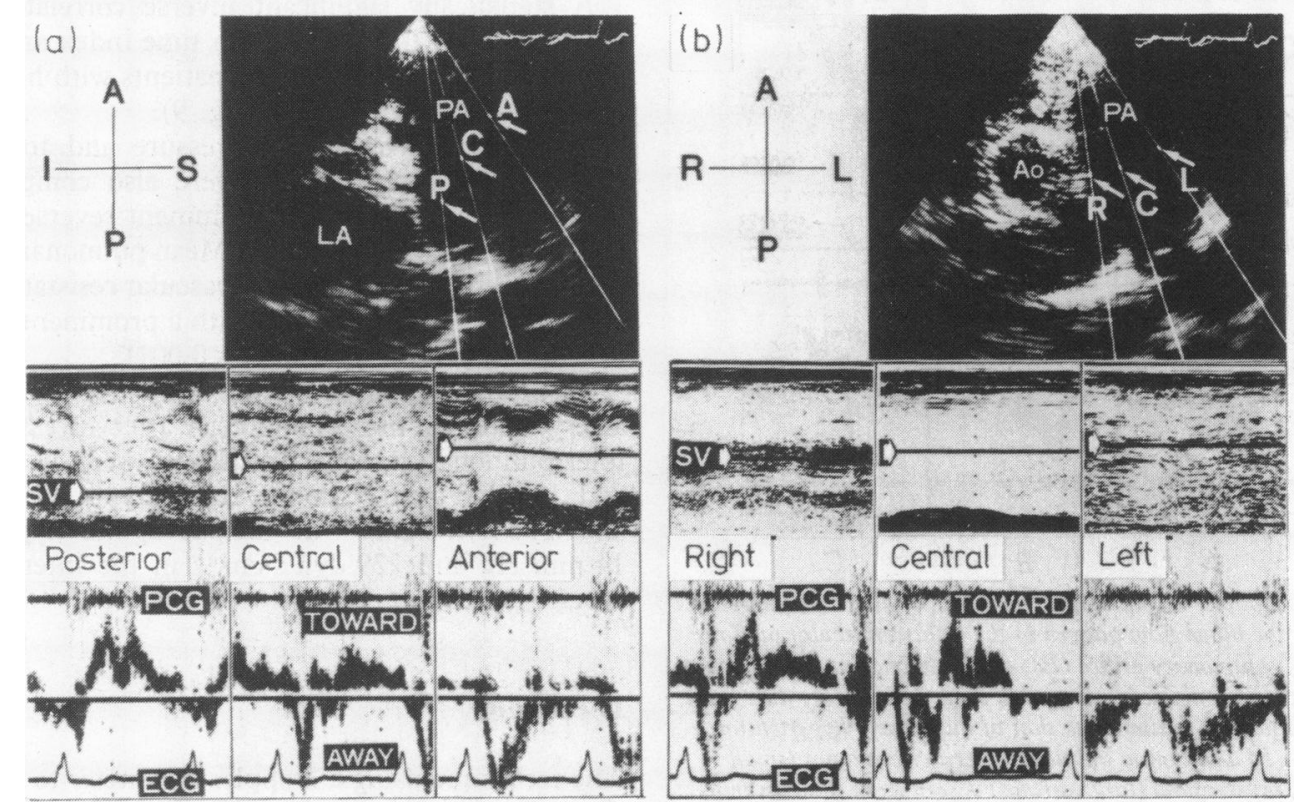

Fig. 7 Doppler recording and cross sectional echocardiogram showing sites of reverse flow in the pulmonary trunk in a patient with pulmonary hypertension: (a) sagittal section, (b) horizontal section. Blood flow pattern in the left anterior part of pulmonary trunk is different from that in the right posterior part. In the left anterior part only forward flow (away from the transducer) is seen throughout systole. In contrast, the forwardflow ends in mid-systole and is followed by reverse flow in the right posterior part. Ao, aorta; PA, pulmonary artery; LA, left atrium; $R$, right; $C$, centre; $P$, posterior; $A$, anterior; $L$, left; $L A$, left atrium; $P A$, pulmonary artery. 


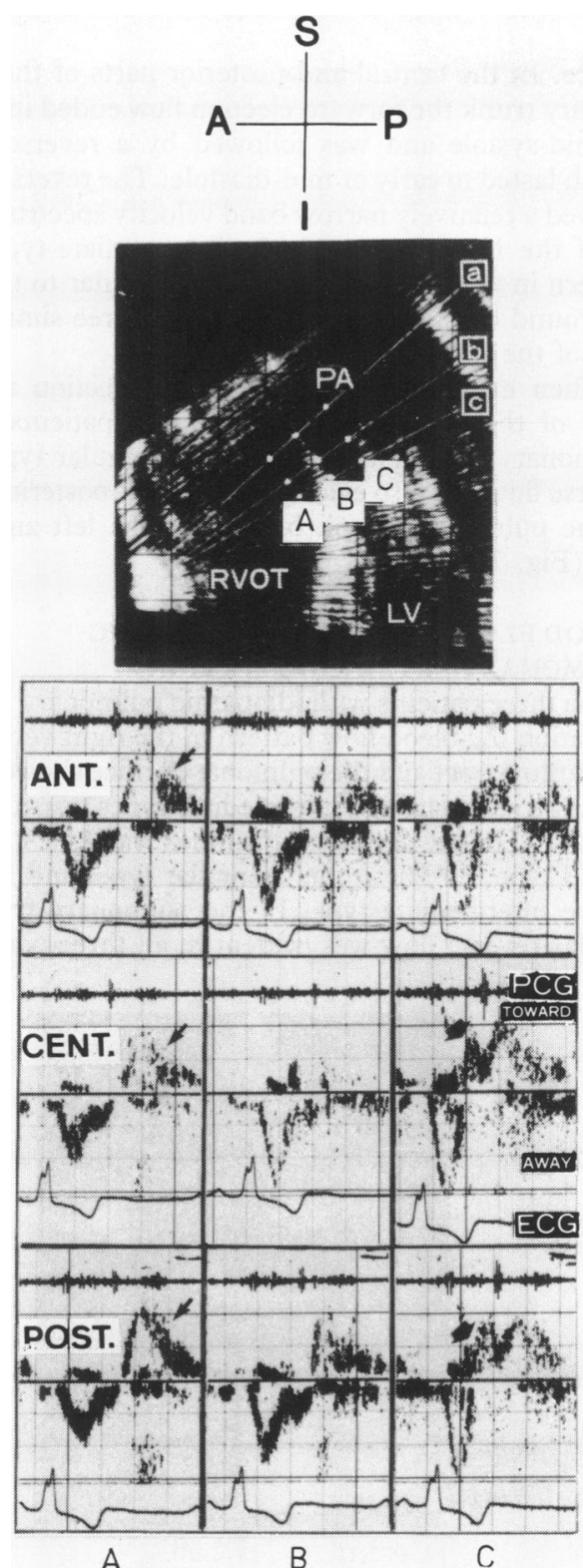

Fig. 8 Doppler recording and cross sectional echocardiogram showing the blood flow pattern in the right ventricular outflow tract $(A)$, pulmonary orifice $(B)$, and pulmonary trunk $(C)$ in a patient with pronounced idiopathic pulmonary artery dilatation. The flow pattern is similar to that in pulmonary hypertension (Fig. 6). In addition to pulmonary regurgitant flows (small arrows), reverse flow (large arrows) is seen as in patients with pulmonary hypertension. The reverse flow appears from mid-systole in the pulmonary trunk, even in its anterior part. In this case, a pulmonary regurgitant signal in the right ventricular oufflow tract begins after the second heart sound, being different from the reverse flow in the pulmonary trunk. RVOT, right ventricular oufflow tract; $P A$, pulmonary artery; $L V$, lefi ventricle; ANT, anterior; CENT, central; POST, posterior.
Okamoto, Miyatake, Kinoshita, Sakakibara, Nimura

RELATION BETWEEN BLOOD FLOW PATTERN AND MEAN PULMONARY ARTERY PRESSURE AND

TOTAL PULMONARY VASCULAR RESISTANCE

In the 50 patients with valvular heart disease and atrial septal defect the blood flow pattern in the pulmonary orifice was examined in relation to mean pulmonary artery pressure and total pulmonary vascular resistance.

Mean pulmonary artery pressure ranged from 8 to $30 \mathrm{~mm} \mathrm{Hg}$ (mean $18.5 \pm 5.1 \mathrm{~mm} \mathrm{Hg}$ ) in the 23 patients with the round type (Table). It was significantly higher in the 14 patients with the triangular type than in those with the round type (range 17$68 \mathrm{~mm} \mathrm{Hg}$, mean $46.6 \pm 17.7 \mathrm{~mm} \mathrm{Hg})(p<0.001)$. Thirteen of the 14 patients with the triangular type had pulmonary hypertension with a mean pulmonary artery pressure $>25 \mathrm{~mm} \mathrm{Hg}$. In the 13 patients with the intermediate type mean pulmonary artery pressure ranged from 18 to $39 \mathrm{~mm} \mathrm{Hg}$ (mean $27.2 \pm 7.2 \mathrm{~mm} \mathrm{Hg}$ ) and appeared to lie between that of the round and triangular types.

Total pulmonary vascular resistance was significantly higher in the 14 patients with the triangular type than in those with the round type $(p<0.001)$ (Table). In the 13 cases with the intermediate type the values lay between those of the round and those of the triangular types (Table).

A statistically significant inverse correlation was noted between the acceleration time index and mean pulmonary pressure in the 50 patients with heart disease $(r=-0.78, p<0.005)$ (Fig. 9).

Mean pulmonary artery pressure and total pulmonary vascular resistance were also compared in patients with and without prominent reverse flow in the pulmonary trunk (Table). Mean pulmonary artery pressure and total pulmonary vascular resistance were significantly higher in those with a prominent reverse flow than in those without $(p<0.001)$.

In the three patients with idiopathic pulmonary artery dilatation mean pulmonary artery pressure and total pulmonary vascular resistance were $10 \mathrm{~mm} \mathrm{Hg}$ and 134 dyn s cm$~^{-5}$ in the round type, $11 \mathrm{~mm} \mathrm{Hg}$ and 238 dyn s cm$~_{-5}$ in the triangular type, and $14 \mathrm{~mm} \mathrm{Hg}$ and 229 dyn s cm${ }^{-5}$ in the intermediate type respectively.

\section{Discussion}

The technique using a Doppler flowmeter to examine the pulmonary area was described by Light using the continuous wave mode. ${ }^{19}$ In some cardiovascular diseases this identified abnormalities in the flow pattern in an area which is difficult to examine with ultrasound.

The combined use of cross sectional echocardiogra- 
Table Pulmonary artery pressure and total pulmonary vascular resistance in relation to blood flow pattern in the pulmonary orifice and presence or absence of prominent reverse flow in the pulmonary trunk

\begin{tabular}{|c|c|c|c|c|c|c|}
\hline & \multirow{2}{*}{$\begin{array}{l}\text { No of } \\
\text { patients }\end{array}$} & \multicolumn{2}{|c|}{ Mean pulmonary artery pressure ( $\mathrm{mm} \mathrm{Hg}$ ) } & \multicolumn{2}{|c|}{ Total pulmonary resistance (dyn $\mathrm{s} \mathrm{cm}^{-5}$ ) } & \multirow[t]{2}{*}{ Probability } \\
\hline & & Range & Mean $\pm S D$ & Range & Mean $\pm S D$ & \\
\hline $\begin{array}{l}\text { Flow pattern: } \\
\text { Round } \\
\text { Triangular } \\
\text { Intermediate }\end{array}$ & $\begin{array}{l}23 \\
14 \\
13\end{array}$ & $\begin{array}{r}8-30 \\
17-68 \\
18-39\end{array}$ & $\begin{array}{l}18 \cdot 5 \pm 5 \cdot 1 \\
46 \cdot 6 \pm 17 \cdot 7^{\star \star} \\
27 \cdot 2 \pm 7 \cdot 2^{\star \star}\end{array}$ & $\begin{array}{l}41-489 \\
119-2176 \\
117-835\end{array}$ & $\begin{array}{c}170 \pm 119 \\
1046 \pm 601^{\star \star} \\
571 \pm 234^{\star}\end{array}$ & $\begin{array}{l}<0.001^{\star \star} \\
<0.05^{\star}\end{array}$ \\
\hline $\begin{array}{l}\text { Reverse flow: } \\
\text { Absent } \\
\text { Present }\end{array}$ & $\begin{array}{l}24 \\
17\end{array}$ & $\begin{array}{l}12-39 \\
17-68\end{array}$ & $\begin{array}{l}23 \cdot 2 \pm 6 \cdot 7 \\
46 \cdot 7 \pm 16 \cdot 7 \dagger\end{array}$ & $\begin{array}{c}51-835 \\
119-2176\end{array}$ & $\begin{array}{c}361 \pm 269 \\
1038 \pm 567 \dagger\end{array}$ & $<0.001 \dagger$ \\
\hline
\end{tabular}

$\star v$ round type.

$\dagger v s$ absence.

phy and the pulsed Doppler technique enabled us to perform regional measurements of blood flow at multiple sites in the cardiac chambers. The size of the sample volume is generally small in comparison with the size of cardiac chambers. The size of the lumen from the right ventricular outflow tract to the pulmonary trunk is usually $20-25 \mathrm{~mm}$ in diameter in healthy adults and larger in patients with pulmonary hypertension. In the present study, the size of the sample volume was $5 \mathrm{~mm}$ (depth) $\times 6 \mathrm{~mm}$ (width) or $2 \mathrm{~mm}$ (depth) $\times 4 \mathrm{~mm}$ (width). It is small enough to measure the blood flow in separate parts of the pulmonary area. Analysing the blood flow pattern in the pulmonary area at nine points is considered to be reasonable.

The technique using the pulsed Doppler flowmeter has shown that the blood flow pattern in the heart varies greatly with different anatomical lesions. The pattern may be identified as shunt flows ${ }^{4-6}$ in congenital malformations, regurgitant flows ${ }^{7-14}$ in valvu-

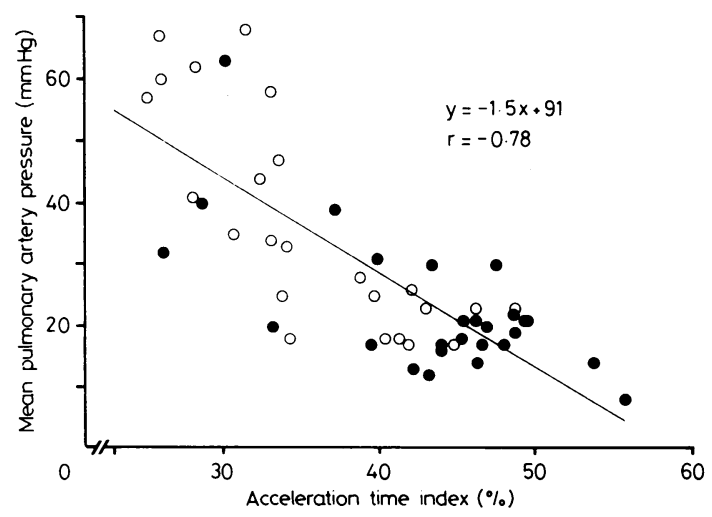

Fig. 9 Relation between acceleration time index and mean pulmonary artery pressure. $(O)$ patients with valvular heart disease; (O) patients with atrial septal defect. lar incompetence, and turbulent flows ${ }^{15-18}$ with high velocity in valvular stenoses. In the present study, the blood flow pattern varied not only with the anatomical lesion but also as a result of a functional event, a pressure change. The flow pattern also differed in different parts of the pulmonary area.

In the present study, the triangular configuration of the flow pattern seemed to be the specific feature of the pulmonary ejection flow in pulmonary hypertension, whereas the round configuration was usually seen in healthy subjects. The triangular pattern in pulmonary hypertension has been also reported using the continuous wave Doppler technique. ${ }^{20}$ In the present study, however, the pulsed wave mode showed that the flow pattern with a reverse component varied at different parts of the pulmonary area. Thus the pulsed wave mode allowed us to analyse the regional haemodynamic conditions in more detail in the pulmonary area than did the continuous wave mode.

Many factors may be responsible for these characteristic features. Firstly, Gleason and Braunwald suggested that right and left ventricular peak $\mathrm{dP} / \mathrm{dt}$ increased in acute pressure overload of the relevant ventricle. ${ }^{21}$ This increase may cause the rapid acceleration of the flow in the pulmonary artery. Secondly, as the abnormal flow pattern was related to the total pulmonary vascular resistance, which was consistent with Hatle and Angelsen's data using the continuous Doppler technique, ${ }^{20}$ the reduced capacitance and the increased impedance of the pulmonary vascular tree in pulmonary hypertension ${ }^{22}$ may be of primary importance in causing deceleration to begin early in the pulmonary flow. This theory is supported by the results of an experimental study, in which pulmonary vasoconstriction induced by serotonin produced sharp peaks in the pulmonary artery flow pulse. ${ }^{23}$ Thirdly, a rapid pulse wave velocity is usually seen in pulmonary hypertension, possibly due to the increased stiffness of the pulmonary artery. ${ }^{24}$ It may cause an earlier 
reflection of a pulsatile wave from the periphery, resulting in the deceleration of the flow beginning early.

This mechanism may also explain the early beginning of deceleration of the pulmonary arterial flow in idiopathic pulmonary artery dilatation, because in this condition-as the pulmonary arterial walls are distended-their reserved capacity of distensibility might be reduced. ${ }^{25}$ Of course, these factors are generally dependent on each other and probably affect the blood flow pattern at the same time.

In pulmonary hypertension and idiopathic pulmonary artery dilatation, a prominent reverse flow occurred after the short forward ejection flow in the pulmonary trunk. Such a prominent reverse flow was not evident in healthy subjects. The reversal of flow began from mid-systole or occasionally even from early systole. These findings seem to indicate a vortex in the right posterior part of the pulmonary trunk (Fig. 10).

It must be considered whether the reverse component might be an artefact. Firstly, the movement of the whole pulmonary artery relative to the transducer may cause an apparent reverse flow in the velocity spectrum as the pulmonary artery moves anteriorly in systole. However, the velocity of the anterior movement of the pulmonary artery does not seem to exceed that of the ejection flow, as is shown on the echocardiogram. Secondly, the pulmonary sinuses of Valsalva may have been sampled. These sinuses would contain vortices in the aorta. The reverse flow in the present study was, however, observed not only in the vicinity of the pulmonary valve but also in the area away from the valve. Furthermore, it was detected in the right posterior part of the pulmonary trunk but not in the left anterior part.

The most likely factors responsible for the reverse flow are the curved path and dilatation of the pulmonary artery. In general, the pulmonary trunk turns posteriorly. The pathway from the pulmonary trunk to the right pulmonary artery shows a more curvilinear route than that to the left pulmonary artery. The curved path of the vessel is enhanced in pulmonary hypertension and idiopathic pulmonary artery dilatation. The dilatation of the pulmonary trunk and the curved path could cause streamline separation ${ }^{26}$ in the right posterior part of the vessel, resulting in vortex formation. This vortex is detected as reverse flow. As mentioned above, the whole pulmonary artery moves anteriorly in systole. If there is a jet from the pulmonary orifice to the pulmonary artery the sample volume may be within the jet in early systole and then within the vortex. This mechanism may be an additional factor in producing an apparent reverse flow.

As reported by us, pulmonary regurgitation is often detected by the Doppler technique in patients with pulmonary hypertension. ${ }^{10}$ The reverse flow related to pulmonary regurgitation may be seen in the right ventricular outflow tract as well as in the pulmonary (a)
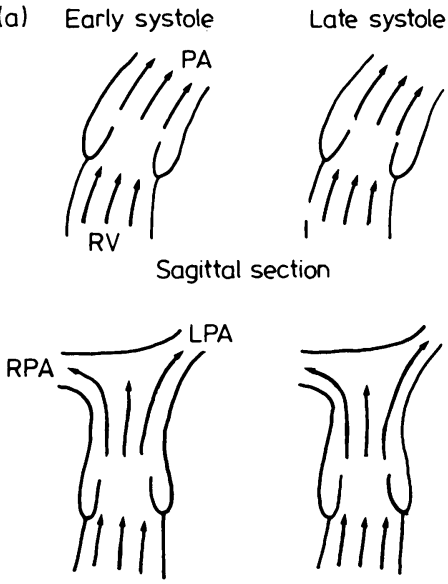

Horizontal section

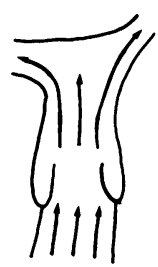

(b)
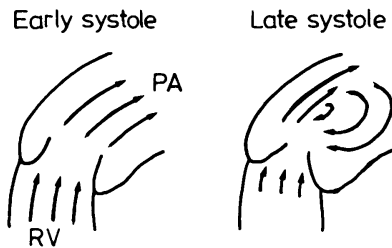

Sagittal section

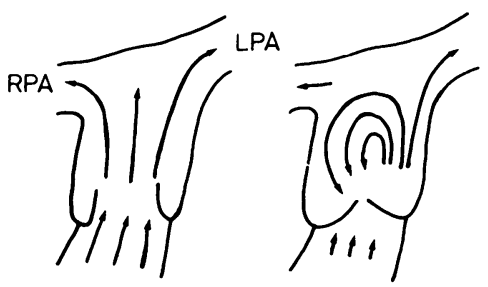

Horizontal section

Fig. 10 Diagram of blood flow in the pulmonary trunk in (a) healthy subjects and (b) patients with pulmonary hypertension and idiopathic pulmonary dilatation. Sagittal section was viewed from the left hand side, and horizontal section from the foot. LPA, left pulmonary artery; $R P A$, right pulmonary artery; $P A$, pulmonary artery; $R V$, right ventricle. Arrows indicate blood flow stream. 
trunk in only diastole, which is different from the reverse flow in the present study. Thus pulmonary regurgitation does not seem to play an important part in the reverse flow formation seen in the present study, even though it might make some contribution in diastole.

In patients with persistent ductus arteriosus Stevenson and coworkers reported that a reverse flow was evident in the pulmonary trunk in diastole, indicating a shunt flow through the ductus. ${ }^{6}$ This flow pattern showed dispersion of the Doppler frequency, producing a harsh audiosignal. Nevertheless, the reverse flow in pulmonary hypertension was different from that in persistent ductus arteriosus. The reverse flow began from mid-systole or even from early systole with a relatively narrow band spectrum of the Doppler frequency.

Thus the reverse flow seen in the present study appears to be one of the specific features of the pulmonary haemodynamics in pulmonary hypertension and idiopathic pulmonary artery dilatation.

This study was supported in part by the Research Grant for Cardiovascular Disease (54-C6) and the Scientific Research Grant (1981) from the Ministry of Health and Welfare of Japan.

\section{References}

1 Johnson SL, Baker DW, Lute RA, Dodge HT. Doppler echocardiography. The localization of cardiac murmurs. Circulation 1973; 48: 810-22.

2 Baker DW, Rubenstein SA, Lorch GS. Pulsed Doppler echocardiography: principles and applications. Am $\mathcal{F}$ Med 1977; 63: 69-80.

3 Kalmanson D, Veyrat C, Bouchareine F, Degroote A. Non-invasive recording of mitral flow velocity patterns using pulsed Doppler echocardiography. Application to diagnosis and evaluation of mitral valve disease. Br Heart f 1977; 39: 517-28.

4 Stevenson JG, Kawabori I, Guntheroth WG. Differentiation of ventricular septal defects from mitral regurgitation by pulsed Doppler echocardiography. Circulation 1977; 56: 14-8.

5 Goldberg SJ, Areias JC, Spitaels SEC, De Villeneuve VH. Use of time interval histographic output from echo-Doppler to detect left-to-right atrial shunts. Circulation 1978; 58: 147-52.

6 Stevenson JG, Kawabori I, Guntheroth WG. Noninvasive detection of pulmonary hypertension in patent ductus arteriosus by pulsed Doppler echocardiography. Circulation 1979; 60: 355-9.

7 Johnson SL. Pulse-Doppler echocardiography. Localization of murmurs and spectrum of clinical applications. In Mason DT, ed. Advances in heart disease, Vol. 1. New York: Grune \& Stratton, 1977: 389-418.

8 Miyatake K, Kinoshita N, Nagata S, et al. Intracardiac flow pattern in mitral regurgitation studied with com- bined use of the ultrasonic pulsed Doppler technique and cross-sectional echocardiography. Am F Cardiol 1980; 45: 155-62.

9. Waggoner AD, Quinones MA, Young JB, et al. Pulsed Doppler echocardiographic detection of right-sided valve regurgitation. Experimental results and clinical significance. Am $\mathcal{Y}$ Cardiol 1981; 47: 279-86.

10 Miyatake $K$, Okamoto $M$, Kinoshita $N$, et al. Pulmonary regurgitation studied with the ultrasonic pulsed Doppler technique. Circulation 1982; 65: 969-76.

11 Miyatake K, Okamoto M, Kinoshita N, et al. Evaluation of tricuspid regurgitation by pulsed Doppler and twodimensional echocardiography. Circulation 1982; 66: 777-84.

12 Veyrat C, Cholot N, Abitbol G, Kalmanson D. Noninvasive diagnosis and assessment of aortic valve disease and evaluation of aortic prosthesis function using echo pulsed Doppler velocimetry. Br Heart f 1980; 43: 393413.

13 Quinones MA, Young JB, Waggoner AD, Ostojic MC, Ribeiro LGT, Miller RR. Assessment of pulsed Doppler echocardiography in detection and quantification of aortic and mitral regurgitation. Br Heart $f$ 1980; 44: 612-20.

14 Ciobanu M, Abassi AS, Allen M, Hermer A, Spellberg R. Pulsed Doppler echocardiography in the diagnosis and estimation of severity of aortic insufficiency. Am $\mathcal{J}$ Cardiol 1982; 49: 339-43.

15 Hatle L, Angelsen BA, Tromsdal A. Noninvasive assessment of aortic stenosis by Doppler ultrasound. $\mathrm{Br}$ Heart $\mathcal{F}$ 1980; 43: 284-92.

16 Young JB, Quinones MA, Waggoner AD, Miller RR. Diagnosis and quantification of aortic stenosis with pulsed Doppler echocardiography. Am f Cardiol 1980; 45: 987-94.

17 Hatle L, Angelsen B, Tromsdal A. Noninvasive assessment of atrioventricular pressure half time by Doppler ultrasound. Circulation 1979; 60: 1096-104.

18 Thuillez C, Théroux P, Bourassa MG, et al. Pulsed Doppler echocardiographic study of mitral stenosis. Circulation 1980; 61: 381-7.

19 Light LH. Initial evaluation of transcutaneous aortovelography. A new non-invasive technique for hemodynamic measurements in the major thoracic vessels. In Reneman R, ed. Cardiovascular applications of ultrasound. Amsterdam \& London: North-Holland, 1974: 325-60.

20 Hatle L, Angelsen BA. Doppler ultrasound in cardiography. Philadelphia: Lea \& Febiger, 1982: 151-5.

21 Gleason WL, Braunwald E. Studies on the first derivative of the ventricular pressure pulse in man. $f$ Clin Invest 1962; 41: 80-91.

22 Reuben SR. Compliance of the human pulmonary arterial system in disease. Circ Res 1971; 29: 40-50.

23 Bergel DH, Milnor WR. Pulmonary vascular impedence in the dog. Circ Res 1965; 16: 401-15.

24 Milnor WR, Conti CR, Lewis KB, O'Rourke MF. Pulmonary arterial pulse wave velocity and impedance in man. Circ Res 1969; 25: 637-49.

25 Bergel DH. The dynamic elastic properties of the arterial wall. F Physiol (Lond) 1961; 156: 458-69.

26 Vennard JK, Street RL. Elementary fluid mechanics. 5th ed. New York: John Wiley, 1975: 313-6. 